\title{
Call for New Approaches for Subcellular Delivery of Anti-cancer Drugs Kan Xiong*
}

Broad Institute of MIT and Harvard, Cambridge, USA

Cancer therapies depend on a precise balance. Malignant cells must be killed while carefully managing collateral damage to normal cells. Though grossly similar in their molecular contents, the tumor cells do differ from their healthy counterparts [1], and anti-cancer therapies take advantage of these differences to selectively interfere with the growth and survival of cancer cells. As our knowledge of cancer biology rapidly expands at the molecular level, particularly on the genetic level, new entry points for cancer therapies emerge. The essential role of genetics in cancer and the need for rapidly dividing cancer cells to avoid DNA damage checkpoints make the genome, its epigenetic state, maintenance, and replication central in cancer.

A major set of cancer therapies focuses on the sensitivity of rapidly dividing cells to DNA damage [2]. Drugs that cause DNA damage or interfere with DNA replication kill rapidly dividing cancer cells, while most of the healthy cells in the body can recover from these insults. Even so, many chemotherapies are so toxic to normal tissues that dosing must be limited to decrease side effects. For early-stage solid tumors, toxicity can be mitigated by traditional tissue-level drug delivery that releases drug molecules at the tumor site [3] However, the standard of care for many solid tumors remains to be systemic application of DNAdamaging chemicals [4-6].

Drugs often exhibit complex effects on cells, even when designed to bind a single target [7] and the toxicity of some cancer therapies to normal cells is often mediated through off-target effects. For example, cisplatin, the mainstay of chemotherapy for many cancers, is known to exert strong effects on targets other than nuclear DNA and exhibit nephrotoxicity, neurotoxicity, and ototoxicity, among other side effects. One concern in retargeting chemotherapeutics to reduce harm to normal tissue is that the efficacy against cancer cells may be reduced. As a result, it is desirable to increase potency for the intended (tumorspecific) target when modifying therapy to mitigate toxicity mediated by off-target effects in normal tissues. Another example is cardio toxicity discovered in trials with HDAC inhibitors attributed to action against the $\mathrm{h}$ ERG potassium channel [8]. That toxicity is sometimes mediated through a different mechanism in a different subcellular compartment in healthy tissues constitutes a new opportunity for drug delivery, one that operates at the cellular level rather than the tissue level. If we can target anti-cancer drugs like DNA damaging agents to their targets in the nucleus, we can maintain or enhance the therapeutic benefit while limiting off-target effects in other compartments of normal cells. $77 \%$ of human proteins are estimated to be localized outside the nucleus according to the Loc DB project; eliminating these as potential mediators of toxicity in normal tissues represents a major step forward in cancer therapy. In addition to above off-target effects, another as critical issue is the development of drug resistance over the course of chemotherapies. Among several known resistance mechanisms, two directly relate to intracellular transportation of drug molecules. One is that the resistant cells decrease or shut down expression of receptors that are essential for cellular uptake of some drug molecules and the other one is that resistant cells express efflux pumps to pump out intracellular drug molecules. Both mechanisms decrease drug concentration in the cytosol to below the effective level.
In parallel with designing/screening new molecules for different cellular targets, developing efficient and reliable delivery method is a very active research area nowadays. One successful drug delivery approach will likely apply to multiple drug molecules that exhibit similar pathways to their targets in the body. This can significantly reduce the cost for developing new cancer therapies. In fact, numerous novel delivery methods by utilizing nanoparticles (made from polymers, proteins, micelles, lipids, viruses, organometallic compounds, etc), polymeric micelles [9], and antibody/ligand/liposomal / lipid/ cell penetration peptide conjugatic $[10,11]$ have been reported, and some of these have resulted in approved therapies or potential therapies in clinical trials. These new approaches enhance drug potency by increasing cellular uptake, assisting drug molecules to locate their molecular targets (thus increasing efficacy while reducing off-target effects), or increasing drug flux into resistant cells. With a keen interest in developing novel drug. delivery approaches, cancer therapy is entering a new paradigm.

\section{References}

1. Hanahan D, Weinberg RA (2011) Hallmarks of cancer: the next generation Cell $144: 646-674$.

2. Hirsch J (2006) An anniversary for cancer chemotherapy JAMA: The Journal of the American Medical Association 296: 1518-1520.

3. Vasir JK, Labhasetwar $\vee(2005)$ Targeted drug delivery in cancer therapy Technology in cancer research \& treatment 4: 363-374.

4. Chan SL, Mo FKF, Johnson PF, Hui EP, Brigette BY, et al. (2009) New utility of an old marker: serial $\alpha$-fetoprotein measurement in predicting radiologic response and survival of patients with hepatocellular carcinoma undergoing systemic chemotherapy. Journal of Clinical Oncology 27: 446-452.

5. Goffin J, Lacchetti C, Ellis PM, Ung YC, Evans WK, et al. (2010) First-line systemic chemotherapy in the treatment of advanced non-small cell lung cancer: a systematic review. Journal of Thoracic Oncology 5: 260-274.

6. Chidambaram M, Manavalan R, Kathiresan K (2011) Nano therapeutics to overcome conventional cancer chemotherapy limitations. Journal of Pharmacy \& Pharmaceutical Sciences $14: 67-77$.

7. Eugen L, Michael JK, Steven W, Dmitri M, Jacques H, et al. (2012) Largescale prediction and testing of drug activity on side-effect targets. Nature 486 : 361-367.

8. Gryder BE, Sodji QH, Oyelere AK (2012) Targeted cancer therapy: giving histone deacetylase inhibitors all they need to succeed. Future Medicinal Chemistry 4: 505-524.

9. Gong J, Chen M, Zheng Y, Wang S, Wang Y, et al. (2012) Polymeric micelles drug delivery system in oncology. J Control Release 159: 312-323.

*Corresponding author: Kan Xiong Broad Institute of MIT and Harvard, Cambridge, MA, USA, Tel: 02142 ; E-mail: kanxiong@mit.edu

Received November 04, 2014; Accepted November 05, 2014; Published November 12, 2014

Citation: Xiong K (2014) Call for New Approaches for Subcellular Delivery of Anticancer Drugs. J Biomol Res Ther 3: e138. doi: 10.4172/2167-7956.1000e138

Copyright: (c) 2014 Xiong K. This is an open-access article distributed under the terms of the Creative Commons Attribution License, which permits unrestricted use, distribution, and reproduction in any medium, provided the original author and source are credited. 
Citation: Xiong K (2014) Call for New Approaches for Subcellular Delivery of Anti-cancer Drugs. J Biomol Res Ther 3: e138. doi:10.4172/2167$7956.1000 \mathrm{e} 138$

Page 2 of 2

10. Rajendran L, Udayar V, Goodger ZV (2012) Lipid-anchored drugs for delivery into subcellular compartments. Trends Pharmacol Sci 33: 215-222.
11. Stewart KM , Horton KL, Kelley SO (2008) Cell-penetrating peptides as delivery vehicles for biology and medicine. Org Biomol Chem 6: 2242-2255. 